\title{
Varying the performance of helical auxetic yarns by altering component properties and geometry
}

\author{
G.H. Zhang*, O. Ghita, C.P. Lin, K.E. Evans \\ College of Engineering, Mathematics and Physical Sciences, University of Exeter, North Park Road, Exeter EX4 4QF, UK \\ ${ }^{*}$ Corresponding author. guanhuazhang1@gmail.com (G.H.Zhang). Tel: +44 (0) 1392263617.
}

\begin{abstract}
This paper presents a systematic study of the helical auxetic yarn (HAY) via careful in-house fabrication and characterisation of a wide range of polymeric fibres and yarns. It provides a better understanding of the auxetic behavior of the HAY in order to tailor their properties for specific applications. The study focused on three parameters: component moduli, the core/wrap diameter ratio and the initial wrap angle. The results show that a larger difference in component moduli, a higher core/wrap diameter ratio and a lower initial wrap angle can produce a larger maximum negative Poisson's ratio value and thereby a better auxetic performance for HAYs. All three parameters could be carefully utilised when in combination to achieve the required auxetic behaviour of HAYs. Moreover, the instantaneous true Poisson's ratio analysis accurately presents the instantaneous behaviour of highly strain dependent HAYs.
\end{abstract}

Keywords: Auxetic yarn, fibres, extrusion, true strain, strain dependent 


\begin{tabular}{|ll|}
\hline Nomenclature & \\
$\varepsilon_{x}$ & engineering longitudinal strain \\
$\varepsilon_{y}$ & engineering transverse strain \\
$\varepsilon_{x}^{i n t}$ & instantaneous true longitudinal strain \\
$\varepsilon_{y}^{i n t}$ & instantaneous true transverse strain \\
$v$ & Poisson's ratio \\
$v_{x y}$ & engineering Poisson's ratio \\
$v_{x y}^{i n t}$ & instantaneous true Poisson's ratio \\
$\theta$ & cyclic pitch of wrap fibre (m) \\
\hline
\end{tabular}

\section{Introduction}

In comparison to convectional materials, auxetic materials exhibit a negative Poisson's ratio, they expand laterally when stretched and contract laterally when compressed $[1,2]$. Auxetic materials are of interest because of their capability to enhance mechanical properties such as shear modulus, indentation resistance, and fracture toughness [3]. As a consequence, they have potential in many practical applications such as impact absorbing foams, fasteners, composites, auxetic textiles for military use, sandwich panels for aircraft, biomedical and nanotechnology applications [3-8].

The helical auxetic yarn (HAY) [9] is a novel structure comprised of two components: an elastic core and a stiff wrap in the form of a helically wound structure, see Fig. 1. When a tensile load is applied the yarn becomes effectively wider, as the wrap straightens out and 
displaces the core, causing a lateral expansion of the core, and thereby exhibiting an auxetic effect. This type of structure has been considered for several applications, such as healthcare [10], body armour [9], blast curtains, and filtration [11]. According to previous studies [1215], the auxetic behaviour of the HAY can be carefully controlled by selecting fibre diameters, component modulus, the initial geometry and also the applied strain. However, to date, the manufacture and characterisation of the HAY was limited to a narrow range of candidate core fibres, which did not enable the maximum advantage of the auxetic behavior effect. Hence, only a narrow diameter range has been investigated in the previous studies [1217]. This paper describes the expansion, optimisation and tailoring of the utilisation of the HAY through careful in-house fabrication and characterisation of a wide range of polymeric fibres and yarns. We focus here on the variation in component modulus, the core/wrap diameter ratio and the initial wrap angle on the auxetic behaviour of the HAY.

\section{Methods}

Extrusion was employed to fabricate cores of varying diameters. Elastollan ${ }^{\circledR}$ Thermoplastic polyurethane (TPU) - CA85A granules (polyester-based TPU) were purchased from BASF to fabricate monofilament core fibre. Monofilament core fibres with various diameters were fabricated using a Rondol (www.rondol.com) $18 \mathrm{~mm}$ diameter bench top single screw extruder (model-Linear 18). Ultra-high-molecular-weight polyethylene (UHMWPE) fibre and stainless steel wire utilised as the wrap fibres due to their high strength and modulus, were sourced from Monofil Technik. Accurate diameters of core and wrap fibres were measured by optical stereo microscope, see Table 1. Surface morphology of failed HAYs were characterised by Dino-Lite Pro (HR AD7013MZT) digital microscope and scanning electron microscope (SEM) (Hitachi S-3200N). 
Helical auxetic yarns were fabricated using a bespoke spinner, which was developed and described in the previous study [13]. Typical properties for fibres and HAYs are presented in Table 1. Three types of monofilament core fibres and two types of wraps were utilised to manufacture HAYs. The wrap angle was only varied while using the largest core fibre to fabricate HAYs.

Tensile measurements of monofilament core, wrap fibres and HAYs were performed using previous described methods, and engineering longitudinal strain measurements of monofilament core fibres and HAYs were also computed using a previous described image analysis method [13]. Engineering transversal strain measurements of monofilament fibres and HAYs were carried out automatically with the assistance of image processing implemented in MatLab R2011b. For each image, the sample object (the ensemble of the core and wrap) is identified via Sobel edge detection [18] (which creates an image with an emphasis of edges and transitions), followed by morphological operations (dilate, fill and erode). This identification gives a binary image; one colour for the object and the other for the background. The upper and lower boundary curves of the resulting binary image are smoothed using a moving average filter. Local peaks of the upper smoothed boundary, and troughs of the lower smoothed boundary are then identified. To measure the time dependent transversal strain, a pair of local peaks and troughs is chosen at the first image to be that in the oscillation cycle on the wrap near the right end of the HAY. The transversal strain is then calculated as the absolute difference between that local peak and trough in the transverse direction. For the subsequent transversal strain measurements, a series of pairs of local peaks and troughs is determined in such a way that the pair in the current image is closest to that in its previous image along the pulling direction, except at a critical time point where the pair is updated to be on the core near its previous position. Thus pairs of peaks and troughs before 
the critical time are on the wrap, while pairs after the critical time are on the core. This image processing gives a series of transversal strains from a series of images automatically.

Finally, the engineering Poisson's ratio for all samples was calculated using measured engineering strains $\varepsilon_{y}$ and $\varepsilon_{x}$.

$v_{x y}=-\frac{\varepsilon_{y}}{\varepsilon_{x}}$

However, the Poisson's ratio is a measure of instantaneous behaviour and it is not possible to accurately represent the instantaneous behaviour of a highly nonlinear material by using the ratio of engineering stains [19]. Therefore, in this study the instantaneous true Poisson's ratio was calculated by taking local tangents from true strain-true strain graphs. The procedure for computing the instantaneous true Poisson's ratio of the HAY is described in detail in a previous study [20].

$$
v_{x y}^{i n t}=-\frac{\varepsilon_{y}^{i n t}}{\varepsilon_{x}^{i n t}}
$$

\section{Results and discussion}

The Young's moduli of samples A to E were calculated using the small strain region (0.05$0.25 \%$ [21]), see Table 1 . All of the HAYs manufactured and tested in this work failed due to failure of the wrap fibre. Surface morphology of failed HAYs is shown in Fig. 2. Fig. 2a-d shows the core indented by the UHMWPE wrap fibre after the HAY was tested with large strain deformations. A more pronounced core indentation effect is observed when the stiffer stainless steel wrap is employed, see Fig. 2e-g. The SEM images of sample K and O provide more details of the core indentation effect as shown in Fig. 3. As proposed in a previous study [15], the core indentation effect can cause a reduction in the negative Poisson's ratio, 
by reducing the lateral displacement of the core and thereby a decrease in the auxetic performance of the HAY.

Engineering stress-strain curves for HAYs with various core/wrap diameter ratios and initial wrap angles are shown in Fig. 4. Three yarn samples were tested for each combination, and the Young's modulus of all HAYs was calculated using these stress-strain curves. The variations in the stress-strain curve for HAYs are mainly due to sample preparation and experimental errors. Fig. 4a and b indicate that the HAY with a smaller core/wrap diameter ratio is shown to have a higher tensile strength. Variation in the initial wrap angle shows less impact on the tensile strength of the HAY compared to the core/wrap diameter ratio, however it has a significant impact on the elongation of the HAY, see Fig. 4c and d. Table 1 indicates that the tensile strength of UHMWPE wrap is larger than that of stainless steel wrap, and this leads to HAYs with UHMWPE wrap having a higher tensile strength than HAYs with stainless steel wrap as shown in Fig. $4 \mathrm{~b}$ and c. Fig. 4e shows the effect of wrap material on the energy absorption of the HAY and it indicates that the HAY with stainless steel wrap (sample L) is able to absorb more energy than the HAY with UHMWPE wrap (sample F). Fig. 4f demonstrates that sample $\mathrm{O}$ with a higher initial wrap angle absorbs more energy. The variation of the core/wrap diameter ratio on the energy absorption of the HAY is presented in Fig. $4 \mathrm{~g}$ and it indicates that the HAY with a lower core/wrap diameter ratio (sample F) is able to absorb more energy. It is also interesting to note that multifilament wrap fibre is able to spread the load during the tensile test, and cause less core indentation effect to the HAY in comparison with stainless steel wrap, see Fig. 2 and 3.

Figure 5 shows instantaneous true Poisson's ratio analysis of the HAY with TPU core and stainless steel wrap. The instantaneous true $v$ of two monofilament TPU core fibres shows a computed value in the range of $0-2.5$ over a strain of 0.35 . As shown in Fig. 5a, the instantaneous true $v$ of the HAY with $1302.1 \mu \mathrm{m}$ core (sample M) has a rapid decrease at 
very low strains of 0.015 . However, the instantaneous true $v$ of the HAY with $394.5 \mu \mathrm{m}$ core (sample L) has a sharp increase at the beginning followed by a rapid decrease until it reaches the maximum negative value at strains of 0.1 . A sharp increase in $v_{x y}^{\text {int }}$ at low strains is mainly due to the internal helix diameter of the wrap fibre conforming to the unstrained diameter of the core and hence a rapid decrease in the net width of the yarn [13]. A sharp increase in $v_{x y}^{\text {int }}$ does not appear for sample $\mathrm{M}$, due to early activation of the auxetic effect. When computed $v_{x y}^{\text {int }}$ of HAYs reaches its maximum negative value, it starts to increase gradually and approaching zero and remains negative until the failure of the wrap. It is also interesting to note that the activation of true auxetic behaviour for HAY with larger core/wrap diameter ratio is much earlier than the HAY with smaller core/wrap diameter ratio. In addition, the instantaneous true $v$ of the HAY with larger core/wrap diameter ratio reaches its maximum negative value is also much earlier than the HAY with smaller core/wrap diameter ratio, and the maximum negative $v_{x y}^{\text {int }}$ is also higher $(-14.38$ vs -12.21$)$, resulting in a better auxetic performance. Rapid activation of true auxetic behaviour of the HAY could be a great advantage in certain applications.

Fig. $5 \mathrm{~b}$ shows the variation of the instantaneous true $v$ as a function of strain for HAYs with the same core/warp diameter ratio and various initial wrap angles. It indicates that the HAY with lower initial wrap angle has an earlier activation and a better auxetic behaviour. This aligns well with previous studies $[13-15,17]$ when only the engineering $v$ was considered. Sample $\mathrm{N}$ with a $20^{\circ}$ initial wrap angle presents an initial positive increase in $v_{x y}^{\text {int }}$ followed by a sharp decrease to its maximum negative value at a strain of 0.04 . The sharp decrease of $v_{x y}^{i n t}$ for sample $\mathrm{N}$ at a strain of 0.04 is mainly caused by a sudden change in the net width of the HAY from decreasing to increasing, resulting a lateral expansion of the HAY. Similar 
phenomena have been observed for some HAYs in the previous study and it has been well discussed in detail using original dimension data [20].

According to the previous study [13], variation in core/wrap diameter ratio showed less impact on the auxetic performance of a HAY. However, the previous study was limited in choices of fibres, only the wrap fibre was varied. In this work, monofilament stainless steel wrap has been replaced by multifilament UHMWPE wrap and three TPU core fibres of diameters $394.5 \mu \mathrm{m}, 683.6 \mu \mathrm{m}$, and $1302.1 \mu \mathrm{m}$ were employed to fabricate HAYs. The instantaneous true $v$ of these HAYs is shown in Fig. 6. The instantaneous true Poisson's ratio curve of HAYs with $683.6 \mu \mathrm{m}$ (sample G) and $1302.1 \mu \mathrm{m}$ (sample H) TPU core present very similar trends, see Fig. 6a. Both HAYs trigger auxetic behaviour almost at the same time, at a strain of 0.04 however, the HAY with $1302.1 \mu \mathrm{m}$ TPU core has a larger maximum negative $v_{x y}^{\text {int }}$. As expected, the HAY with $394.5 \mu \mathrm{m}$ TPU core (sample F) has a late activation of the auxetic effect compared to other two HAYs and it has the smallest maximum negative $v_{x y}^{\text {int }}$ in this group. Once again, these phenomena demonstrate that a higher core/wrap diameter ratio provides a better auxetic performance of a HAY. Fig. $6 \mathrm{~b}$ shows the variation of the instantaneous true $v$ as a function of strain with the same TPU core and UHMWPE wrap and various initial wrap angles. All of the HAYs have the initial positive $v_{x y}^{\text {int }}$ versus strain followed by decreasing to negative value, and then remain negative, and finally approaching zero until the failure of the wrap. It shows that variation in the initial wrap angle has a great impact on the auxetic performance of the HAY. These results are demonstrated that by Figs. $5 b$ and $6 b$.

Variations in component modulus and core/warp diameter ratio on the auxetic performance of HAYs are presented in Fig. 7. It demonstrates that HAYs could be tailored using these two parameters to provide significantly large maximum negative Poisson's ratio. 
Figure 8 compares the instantaneous true and engineering $v$ for HAYs. The Poisson's analysis for the HAY with UHMWPE wrap and the smallest core/wrap diameter ratio (sample F) is shown in Fig. 8a, the engineering $v$ remains positive until the failure of the wrap; however, the instantaneous true $v$ approaches the zero-crossing at a strain of 0.075 and remains negative until the failure of the wrap. The results for the HAY with stainless steel wrap and the smallest core/wrap diameter ratio (sample L) is presented in Fig. 8d, the engineering $v$ becomes negative at a strain of 0.08 and remains negative until the failure of the wrap while the instantaneous true $v$ crosses the zero line at a strain of 0.06 and remains negative until the failure of the wrap. HAYs with a lower initial wrap angle (samples $\mathrm{H}$ and $\mathrm{M}$ ) trigger the auxetic effect at lower strains, and a higher wrap angle (sample K) enables the auxetic effect at higher strains in both cases of engineering and instantaneous true $v$, see Fig. 8b, c and e. Overall, the instantaneous true and engineering $v$ both have an initial positive $v$, then the instantaneous true $v$ becomes negative while the engineering $v$ remains positive. Finally they both remain negative until the failure of the wrap. The initial positive engineering $v$ of HAYs is caused by the net width of the yarn in the first part of strain ranges is smaller than the starting net width of the yarn. At the engineering activation strain for auxetic effect the net width of the yarn becomes greater than the starting net width of the yarn and then there is a zero-crossing of the engineering $v[12,13]$. The initial positive instantaneous true $v$ is attributed to the net width of the yarn in the first part of strain ranges decreases. At the true activation strain for auxetic behaviour the net width of the yarn starts to increase so that the instantaneous true $v$ becomes negative [20].

The initial wrap angle is a significant parameter that has been employed to tailor the Poisson's ratio of the HAY. Fig. 9 shows the maximum negative instantaneous true and engineering $v$ as a function of the initial wrap angle. TPU core fibre with $1302.1 \mu \mathrm{m}$ diameter was employed for all the HAYs in Fig. 9. Both instantaneous true and engineering maximum 
negative $v$ become less negative with an increase of the initial wrap angle, and HAYs with stainless steel wrap demonstrate a better auxetic performance than that of HAYs with UHMWPE wrap when a lower initial wrap angle is employed. The engineering maximum negative $v$ of the HAY with stainless steel wrap and a $10^{\circ}$ wrap angle shows a large error bar. This behaviour may be attributed to the poor conformance between the core and wrap fibre.

\section{Conclusions}

This paper presents a systematic study of HAYs in terms of their fabrication, characterisation and mechanical properties. This work is the first study presenting a comprehensive auxetic performance analysis of a wide range of core/wrap geometries and properties regarding the instantaneous true and engineering Poisson's ratio of HAYs, and it is also the first use of a stiff stainless steel wire as the wrap. The instantaneous true Poisson's ratio analysis should always be carried out as the engineering Poisson's ratio is misleading in practice. The auxetic performance of HAYs becomes more observable with an instantaneous true Poisson's ratio analysis. Three significant parameters were identified and selected to tailor the auxetic effect: the core/wrap diameter ratio, the initial wrap angle and component moduli. It was found that a larger difference in component moduli, a higher core/wrap diameter ratio and a lower initial wrap angle led to an earlier and better auxetic behaviour, and these parameters should be carefully balanced to tailor the auxetic behaviour of HAYs for a wide range of potential applications.

\section{Acknowledgements}

This work is supported by the UK Engineering and Physical Science Research Council (EPSRC grant No. EP/J004553/1). The authors would like also to acknowledge their colleagues Dave Baker and Yat-Tarng Shyng for technical support. 


\section{References}

1. Lakes, R., Foam Structures with a Negative Poisson's Ratio. Science, 1987. 235(4792): p. 1038-1040.

2. Evans, K.E., Nkansah, M.A., Hutchinson, I.J., and Rogers, S.C., Molecular network design. Nature, 1991. 353(6340): p. 124-124.

3. Evans, K.E. and Alderson, A., Auxetic Materials: Functional Materials and Structures from Lateral Thinking! Advanced Materials, 2000. 12(9): p. 617-628.

4. Lakes, R., Advances in negative Poisson's ratio materials. Advanced Materials, 1993. 5(4): p. 293-296.

5. Yang, W., Li, Z.-M., Shi, W., Xie, B.-H., and Yang, M.-B., Review on auxetic materials. Journal of Materials Science, 2004. 39(10): p. 3269-3279.

6. Assidi, M. and Ganghoffer, J.-F., Composites with auxetic inclusions showing both an auxetic behavior and enhancement of their mechanical properties. Composite Structures, 2012.94(8): p. 2373-2382.

7. Grima, J.N., Cauchi, R., Gatt, R., and Attard, D., Honeycomb composites with auxetic out-of-plane characteristics. Composite Structures, 2013. 106: p. 150-159.

8. Smardzewski, J., Jasińska, D., and Janus-Michalska, M., Structure and properties of composite seat with auxetic springs. Composite Structures, 2014. 113: p. 354-361.

9. Hook, P.B., Evans, K.E., Hannington, J.P., Hartmann-Thompson, C., and Bunce, T.R., Composite Materials and Structures, U.S. Patent No. US2007031667, 2007.

10. Hook, P.B., Composite fibre and related detection system, in U.S Patent No. US20090193906 A12009.

11. Hook, P.B., Uses of Auxetic Fibres, in U.S. Patent No. US20070210011 A12007.

12. Wright, J.R., Sloan, M.R., and Evans, K.E., Tensile properties of helical auxetic structures: A numerical study. Journal of Applied Physics, 2010. 108(4). 
13. Sloan, M.R., Wright, J.R., and Evans, K.E., The helical auxetic yarn - A novel structure for composites and textiles; geometry, manufacture and mechanical properties. Mechanics of Materials, 2011. 43(9): p. 476-486.

14. Wright, J.R., Burns, M.K., James, E., Sloan, M.R., and Evans, K.E., On the design and characterisation of low-stiffness auxetic yarns and fabrics. Textile Research Journal, 2012. 82(7): p. 645-654.

15. Bhattacharya, S., Zhang, G.H., Ghita, O., and Evans, K.E., The variation in Poisson's ratio caused by interactions between core and wrap in helical composite auxetic yarns. Composites Science and Technology, 2014. 102(0): p. 87-93.

16. Miller, W., Hook, P.B., Smith, C.W., Wang, X., and Evans, K.E., The manufacture and characterisation of a novel, low modulus, negative Poisson's ratio composite. Composites Science and Technology, 2009. 69(5): p. 651-655.

17. Miller, W., Ren, Z., Smith, C.W., and Evans, K.E., A negative Poisson's ratio carbon fibre composite using a negative Poisson's ratio yarn reinforcement. Composites Science and Technology, 2012. 72(7): p. 761-766.

18. Parker, J.R., Algorithms for Image Processing and Computer Vision. 1997, New York: John Wiley \& Sons, Inc.

19. Smith, C.W., Wootton, R.J., and Evans, K.E., Interpretation of experimental data for Poisson's ratio of highly nonlinear materials. Experimental Mechanics, 1999. 39(4): p. $356-362$.

20. Zhang, G.H., Ghita, O., and Evans, K.E., The fabrication and mechanical properties of a novel 3-component auxetic structure for composites. Composites Science and Technology, 2015. 117: p. 257-267.

21. ISO, Plastics -- Determination of tensile properties -- Part 1: General principles, 2012. 
$\mathbf{a}$

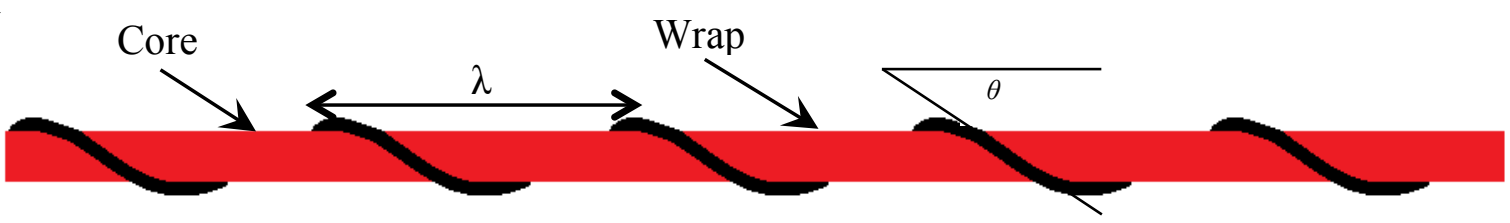

b

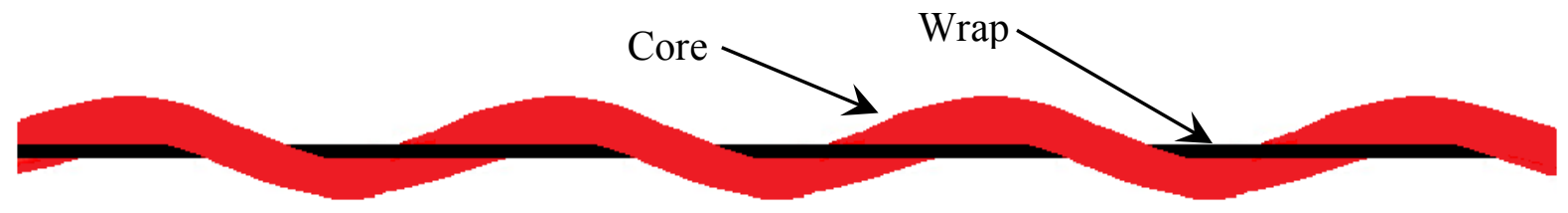

Fig. 1. Illustration of a HAY comprising a core and a helically wound wrap at initial angle $\theta$ : (a) HAY at zero strain and (b) HAY at maximum strain (after [12]). 

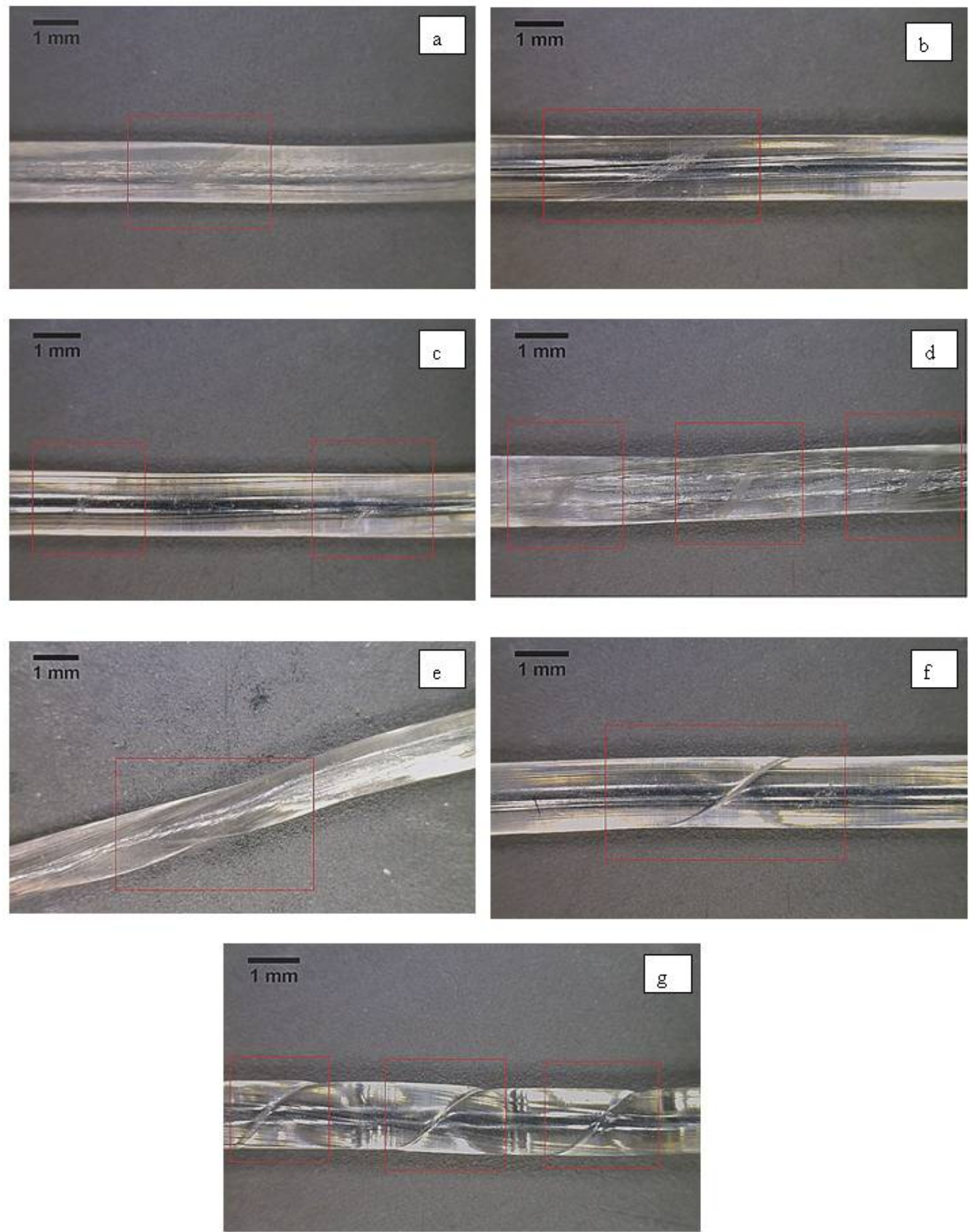

Fig. 2. Surface morphology of failed HAYs obtained by digital microscope: (a) sample H; (b) sample I; (c) sample J; (d) sample K; (e) sample M; (f) sample N; (g) sample O. 

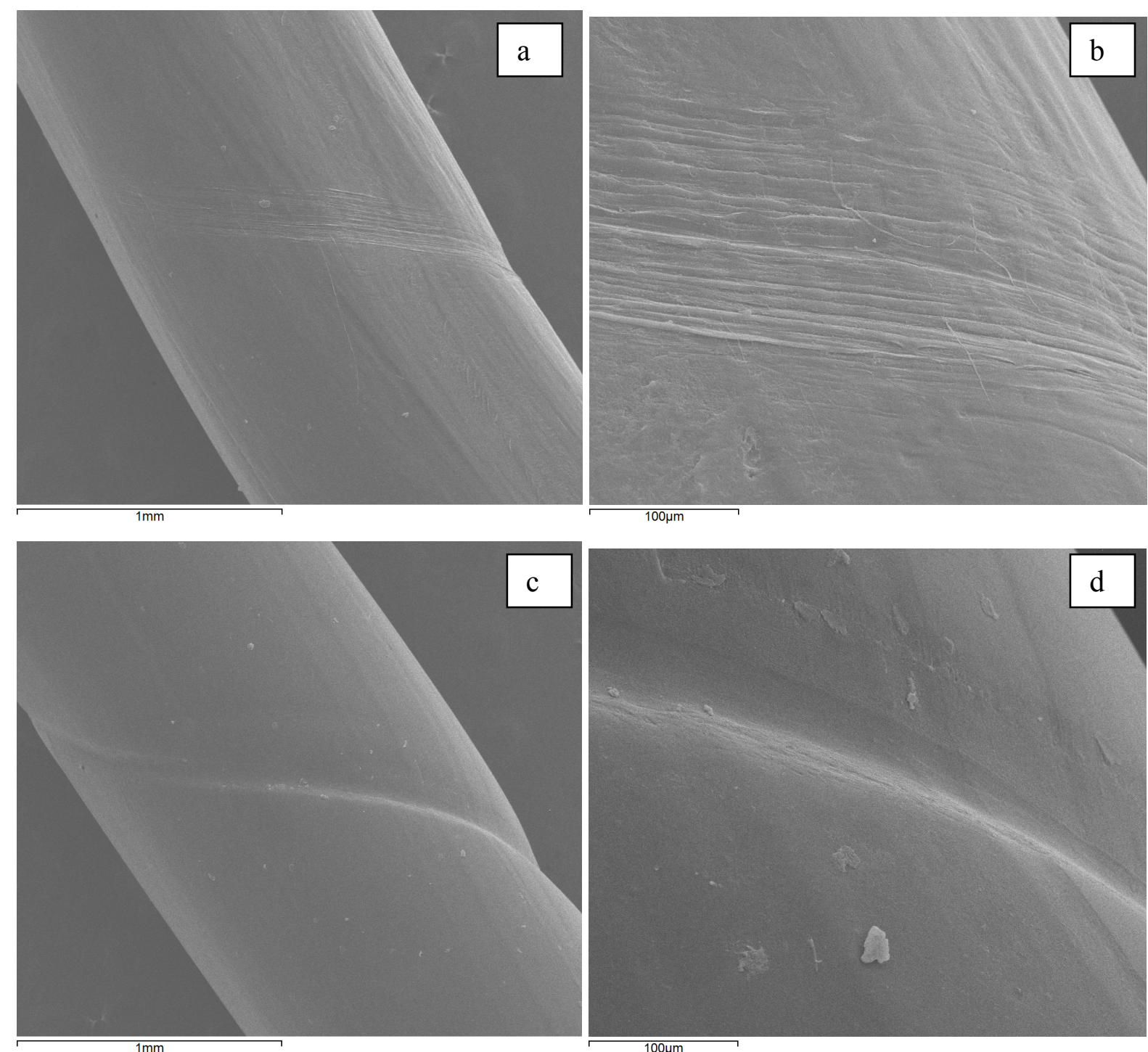

Fig. 3. Surface morphology of failed HAYs obtained by SEM: (a) and (b) sample K; (c) and (d) sample O. 

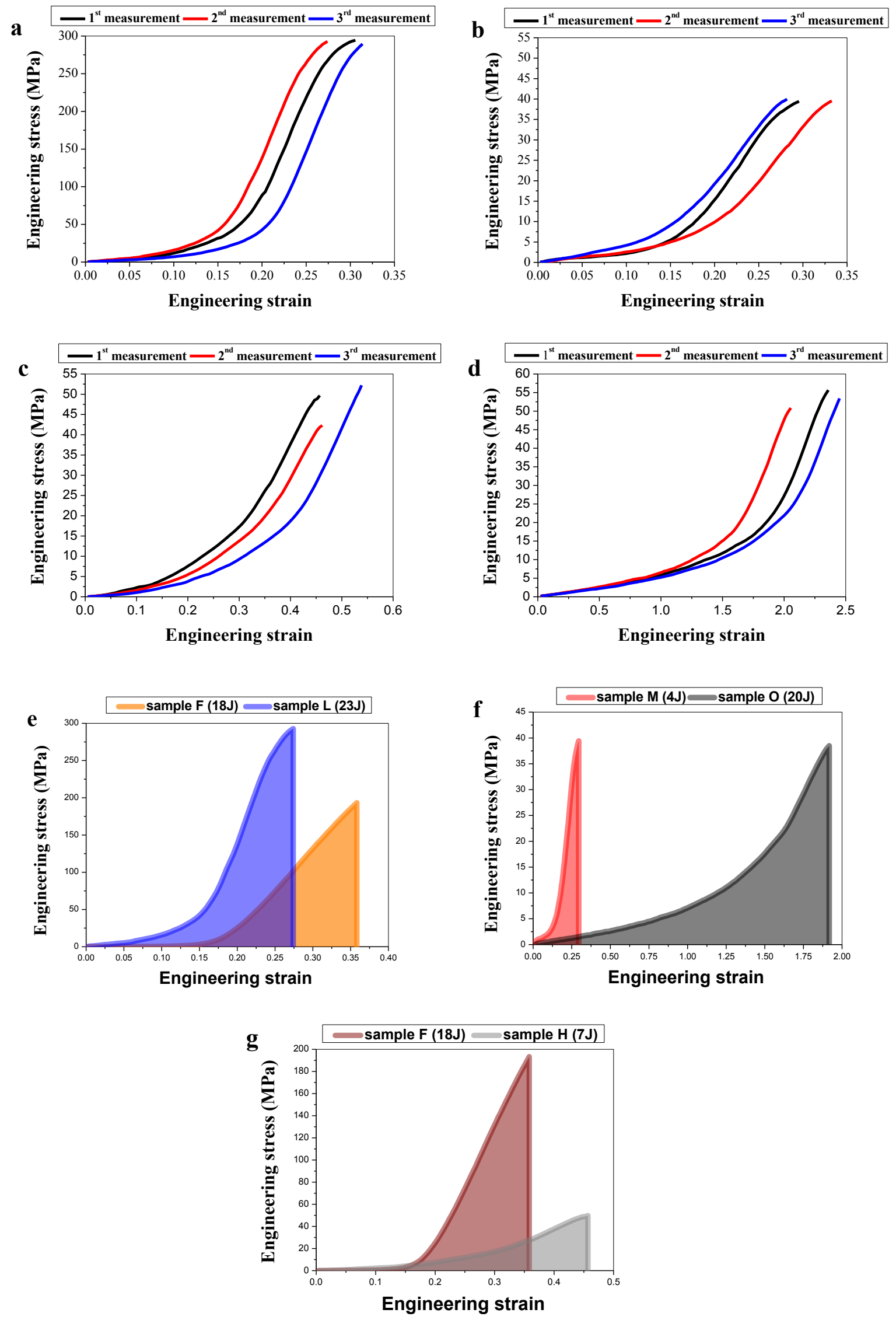

Fig. 4. Stress-strain curve of: (a) sample L; (b) sample M; (c) sample H; (d) sample K; (e) samples F and L; (f) samples $\mathrm{M}$ and $\mathrm{O}$; (g) samples F and H. 
$\mathbf{a}$

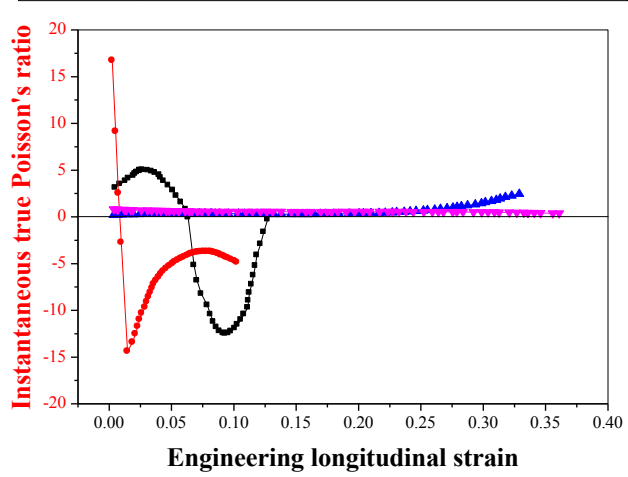

b

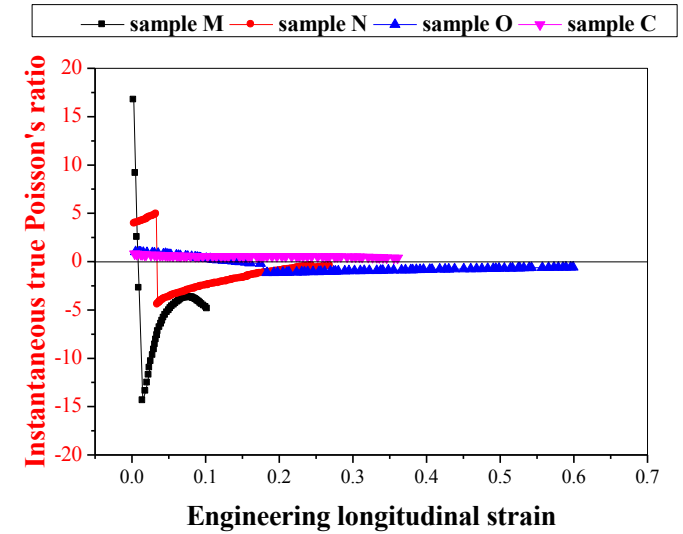

Fig. 5. Instantaneous true Poisson's ratio analysis of HAY with TPU core and stainless steel wrap: (a) various core to wrap diameter ratios; (b) various initial wrap angles.
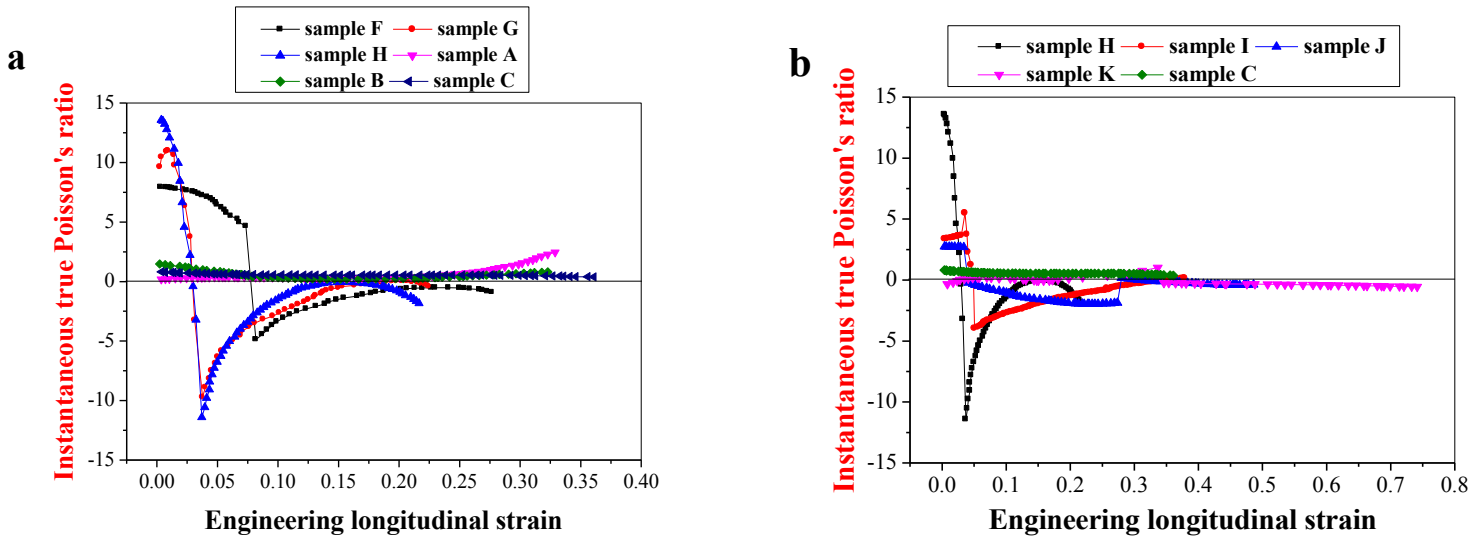

Fig. 6. Instantaneous true Poisson's ratio analysis of HAY with TPU core and UHMWPE wrap: (a) various core to wrap diameter ratios; (b) various initial wrap angles. 


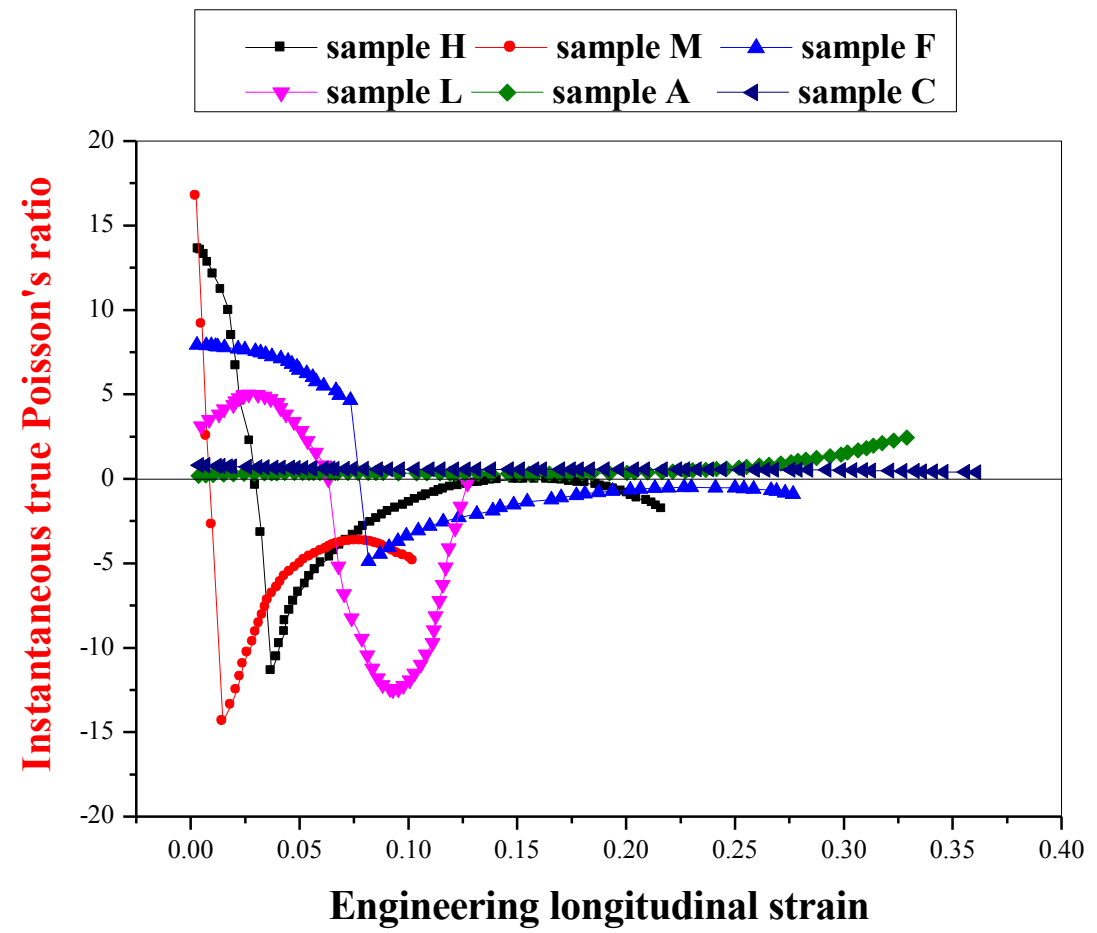

Fig. 7. Comparison of instantaneous true Poisson's ratio analysis between samples. 
$\mathbf{a}$
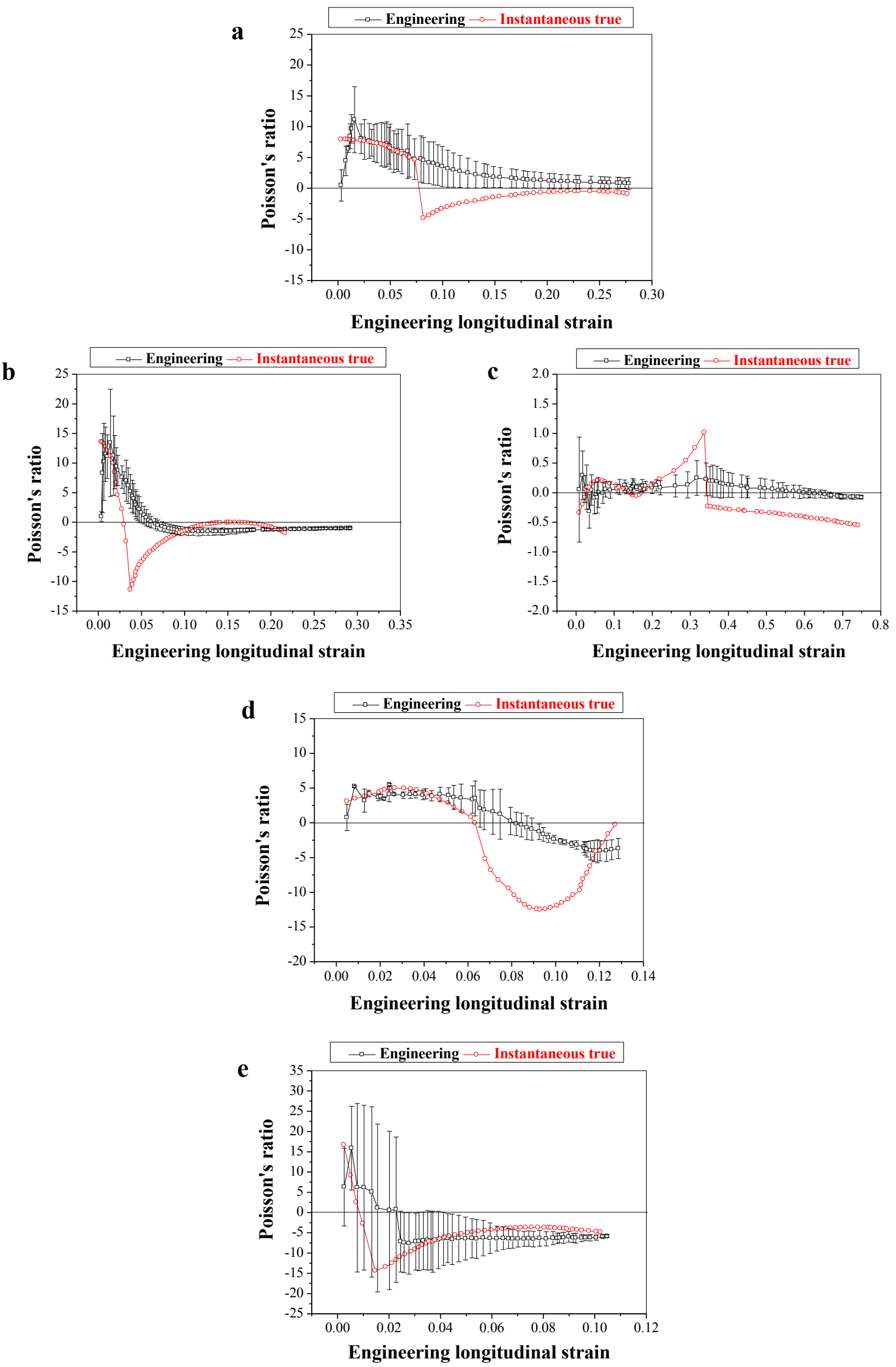

Fig. 8. Comparison of engineering and instantaneous true Poisson's ratio analysis: (a) sample F; (b) sample H; (c) sample K; (d) sample L; (e) sample M. 

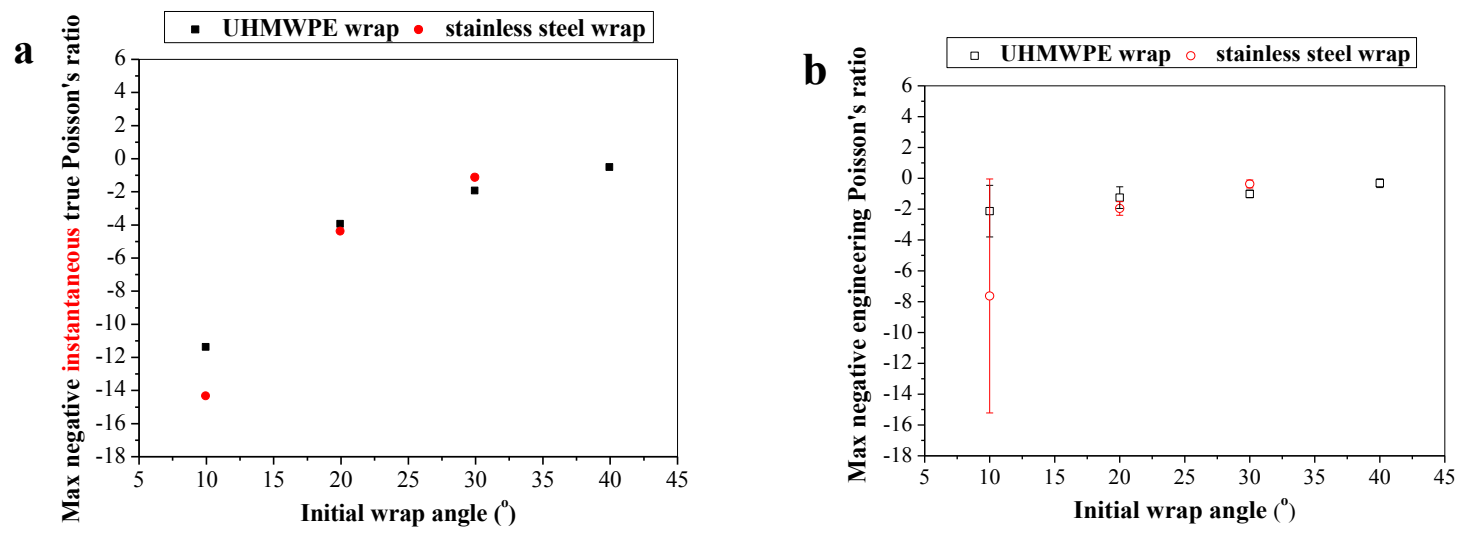

Fig. 9. Maximum negative instantaneous true (a) and engineering (b) Poisson's ratio of HAYs as a function of the initial wrap angle. 
Table 1. Typical properties for fibres and HAYs.

\begin{tabular}{|c|c|c|c|c|c|c|c|}
\hline Sample & Type & $\begin{array}{l}\text { TPU } \\
\text { core } \\
(\mu \mathrm{m})\end{array}$ & $\begin{array}{c}\text { UHMWPE } \\
\text { wrap } \\
(\mu \mathrm{m})(+/- \\
27 \mu \mathrm{m})\end{array}$ & $\begin{array}{c}\text { Stainless } \\
\text { steel wrap } \\
(\mu \mathrm{m})(+/- \\
0.3 \mu \mathrm{m})\end{array}$ & $\begin{array}{c}\text { Initial } \\
\text { wrap } \\
\text { angle } \\
\left(^{0}\right)\end{array}$ & $\begin{array}{c}\text { Young's } \\
\text { modulus } \\
\text { (MPa) }\end{array}$ & $\begin{array}{c}\text { Tensile } \\
\text { strength } \\
\text { (MPa) }\end{array}$ \\
\hline $\mathrm{A}$ & Fibre & $\begin{array}{c}394.5 \pm \\
23.8\end{array}$ & - & - & - & $12.5 \pm 2.2$ & - \\
\hline B & Fibre & $\begin{array}{c}683.6 \pm \\
30.5\end{array}$ & - & - & - & $14.7 \pm 3.6$ & - \\
\hline $\mathrm{C}$ & Fibre & $\begin{array}{c}1302.1 \pm \\
23.4\end{array}$ & - & - & - & $14.8+1.4$ & \\
\hline D & Fibre & - & 370 & - & - & $\begin{array}{c}23,000 \pm \\
3,000\end{array}$ & $\begin{array}{c}4,300 \pm \\
160\end{array}$ \\
\hline E & Wire & - & - & 139.8 & - & $\begin{array}{c}43,000 \pm \\
1,700\end{array}$ & $\begin{array}{c}3,600 \pm \\
100\end{array}$ \\
\hline F & $\begin{array}{l}\text { Helical } \\
\text { auxetic } \\
\text { yarn }\end{array}$ & $\begin{array}{c}394.5 \pm \\
23.8\end{array}$ & 370 & - & $\begin{array}{c}12.8 \pm \\
1.8\end{array}$ & - & - \\
\hline G & $\begin{array}{l}\text { Helical } \\
\text { auxetic } \\
\text { yarn }\end{array}$ & $\begin{array}{c}683.6 \pm \\
30.5\end{array}$ & 370 & - & $\begin{array}{c}12.4 \pm \\
1.4\end{array}$ & - & - \\
\hline $\mathrm{H}$ & $\begin{array}{l}\text { Helical } \\
\text { auxetic } \\
\text { yarn }\end{array}$ & $\begin{array}{c}1302.1 \pm \\
23.4\end{array}$ & 370 & - & $\begin{array}{c}12.5 \pm \\
1.5\end{array}$ & - & - \\
\hline I & $\begin{array}{l}\text { Helical } \\
\text { auxetic } \\
\text { yarn }\end{array}$ & $\begin{array}{c}1302.1 \pm \\
23.4\end{array}$ & 370 & - & $\begin{array}{c}20.6 \pm \\
1.2\end{array}$ & - & - \\
\hline $\mathrm{J}$ & $\begin{array}{l}\text { Helical } \\
\text { auxetic } \\
\text { yarn }\end{array}$ & $\begin{array}{c}1302.1 \pm \\
23.4\end{array}$ & 370 & - & $\begin{array}{c}30.9 \pm \\
1.4\end{array}$ & - & - \\
\hline K & $\begin{array}{l}\text { Helical } \\
\text { auxetic } \\
\text { yarn }\end{array}$ & $\begin{array}{c}1302.1 \pm \\
23.4\end{array}$ & 370 & - & $\begin{array}{c}40.7 \pm \\
1.9\end{array}$ & - & - \\
\hline L & $\begin{array}{l}\text { Helical } \\
\text { auxetic }\end{array}$ & $\begin{array}{c}394.5 \pm \\
23.8\end{array}$ & - & 139.8 & $\begin{array}{c}13.0 \pm \\
0.4\end{array}$ & - & - \\
\hline
\end{tabular}




\begin{tabular}{|c|c|c|c|c|c|c|c|}
\hline & yarn & & & & & & \\
\hline $\mathrm{M}$ & $\begin{array}{l}\text { Helical } \\
\text { auxetic } \\
\text { yarn }\end{array}$ & $\begin{array}{c}1302.1 \pm \\
23.4\end{array}$ & - & 139.8 & $\begin{array}{c}10.9 \pm \\
1.5\end{array}$ & - & - \\
\hline $\mathrm{N}$ & $\begin{array}{l}\text { Helical } \\
\text { auxetic } \\
\text { yarn }\end{array}$ & $\begin{array}{c}1302.1 \pm \\
23.4\end{array}$ & - & 139.8 & $\begin{array}{c}19.8 \pm \\
1.1\end{array}$ & - & - \\
\hline $\mathrm{O}$ & $\begin{array}{l}\text { Helical } \\
\text { auxetic } \\
\text { yarn }\end{array}$ & $\begin{array}{c}1302.1 \pm \\
23.4\end{array}$ & - & 139.8 & $\begin{array}{c}31.4 \pm \\
1.5\end{array}$ & - & - \\
\hline
\end{tabular}

